\title{
Creative Design for Barrier Tolls
}

\author{
Liu Xueying; Fei Xiaoyan; Tao Zejun \\ College of Science \\ Shanghai University, \\ Shanghai, 200444, China \\ 1xyace96@163.com
}

\begin{abstract}
This paper is aimed to design the toll plaza and its merging pattern to relieve the congestion as much as possible. Firstly, we calculate the necessary number of tollbooths for $L$ traffic lanes to avoid jam in the fan-out area is $4 \mathrm{~L}$. Secondly, we want to decide the arranging shape of tollbooths. Compared traditional barrier toll with tollbooths, we are considering another shape to arrange tollbooths to avoid the shortcoming of traditional merging pattern that the overlapping area of neighboring vehicles' driving track often cause crash. Our model includes innovative and pioneering thinking and is especially suitable for autonomous vehicles, which means it will have extensive application in the future.
\end{abstract}

Keywords-Barrier tolls; Avoid congestion; Optimal design of the barrier toll system

\section{INTRODUCTION}

Merging within a toll plaza calls for merging of traffic flow from a higher number of toll egress lanes into a lower number of traffic lanes within a limited space. So the design of the toll plaza and its merging pattern is significantly important to avoid congestion [1-4]. Except considering the accident prevention, throughput and the minimum cost of the toll plaza design, we should also discuss different operation model under various traffic situation to achieve the optimal result.

\section{MODEL DESIGN}

\section{A. Avoid Congestion}

A barrier toll is usually regarded as the bottleneck on highways, however we want to minimize the hindering effect to the traffic flow because of the barrier toll system [5-7]. So an ideal flashed into our mind that if all vehicles spend the same time through the barrier toll and have certain as well as similar speed back to the highway, then the barrier toll system can be ignored to every vehicle. To achieve this effect, the critical points is to control the time every vehicle spent in a barrier toll system and the order they enter the barrier toll should be the same as they leave the toll.

\section{B. The Number of Tollbooths to Avoid Congestion}

First we consider an ideal model that there is only one travel lane. We assume that the average length of a vehicle is $l$, the speed of the vehicle in the fan out area is $\mathrm{V}$, and the average time spending on tollbooths is t.To think about an extreme heavy traffic situation that vehicles enter the fan-out area one by one continuously. In order to avoid congestion the number of tollbooths we need is $\left[\frac{\mathrm{vt}}{\mathrm{l}}\right], \quad$ ( $[\mathrm{a}]$ is a celling function $)()$ when there is only one travel lane.

Next, considering a toll highway having $L$ lanes of travel in each direction, then the number of tollbooths B satisfies the following equation:

$$
\mathrm{B}=\left[\frac{\mathrm{vt}}{1}\right] \mathrm{L}
$$

However, the driving vehicles cannot move on the highways one next to one. The safety distance $D$ must exist. If we consider the vehicle and the safety distance behind the vehicle as a whole, then the above equation is reasonable. But the number of tollbooths should be revised as follow:

$$
\mathrm{B}=\left[\frac{\mathrm{vt}}{1+\mathrm{D}}\right] \mathrm{L}
$$

This is the maximum number of tollbooths, completely avoid the congestion before paying. To be specific, by searching data, we know that the average length of a vehicle is $4.6 \mathrm{~m}$, the speed of the vehicle in the fan out area is $40 \mathrm{~km} / \mathrm{h}$, the safety distance can be $50 \mathrm{~m}$, the average time of collecting toll at a time is $18 \mathrm{~s}$.So we can get the exact relation between $B$ and $L: B=4 L$.

\section{The Merging Pattern}

As to the merging pattern, we will let vehicles pass with group to group. The neighboring group won't meet with each other, because they come in with safety distance which causes time interval. The question is whether this time interval can be maintained after paying. We can ensure that because we have controlled every vehicle spend the same time from entering the fan-out area to having paid. Just like every vehicle's time-line is pushed by same time. The time interval won't change.

For example, a two-lane highway will need 8 tollbooths. At a certain time, two vehicles No. 1, No. 2 as a group come in. Following them immediately come in group No. 3, No. 4 , and then No. 5 , No. 6 , then No. 7 , No. 8 , then No. 9, No. 10 .They are arranged as Figure 1.

Our intention is to make sure that they are leaving in the same order as they came in the toll plaza. So we divide 8 
tollbooths into 4 groups, the front four groups can be allocated to the empty tollbooths, where vehicles No. 1 - No. 8 occupy all tollbooths. But because of the model we built above, the number of tollbooths have ensured that when No. 9, No. 10 arrive at tollbooth, No. 1, No. 2 have already paid and left. SoNO No. 9, No. 10 is supposed to go to No. 1 , No. 2 . In the similar way No. 11, No. 12 should follow No. 3, No. 4 entering the same tollbooths. So we need railing and raising lever to conduct and indicate which tollbooth every vehicle should go to (Figure 2, Figure 3)

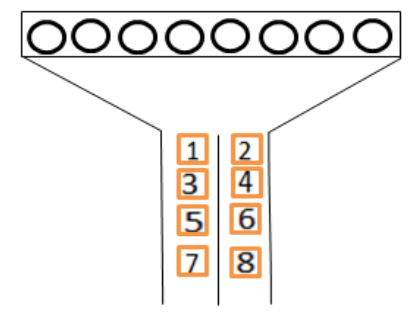

Fig. 1. The original order of traffic flow

Fig. 2. The order of payin

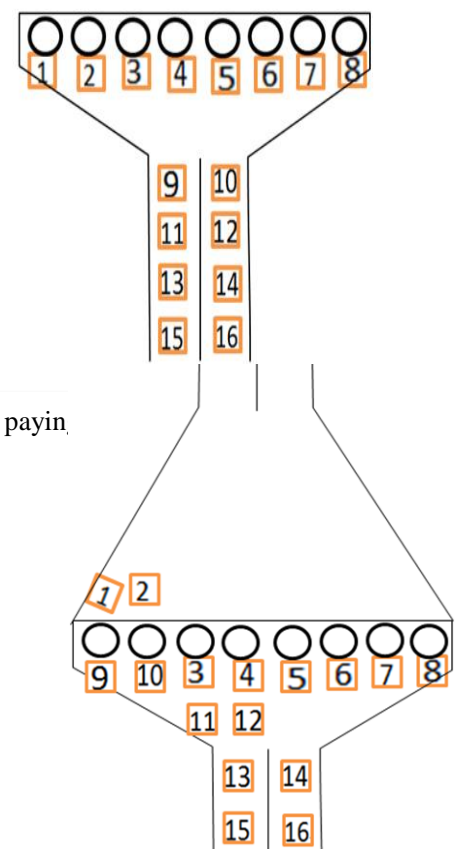

Fig. 3. The order after paying

\section{Distribution of Tollbooths}

Compared with traditional barrier toll with tollbooths placed perpendicular to the direction of traffic flow, we are considering whether there is another shape to arrange tollbooths. Because there are always more tollbooth egress lanes than lanes of traffic, so changing lanes seems inevitable, especially for vehicles getting through the marginal tollbooths. For vehicles need changing lanes, their traveling track must have overlapping area with other vehicles' traveling track which can drive from the tollbooth egress lane to the traffic lane directly. So in the traditional merge pattern the overlapping area increases the potential of accident (Figure 4, Figure 5).

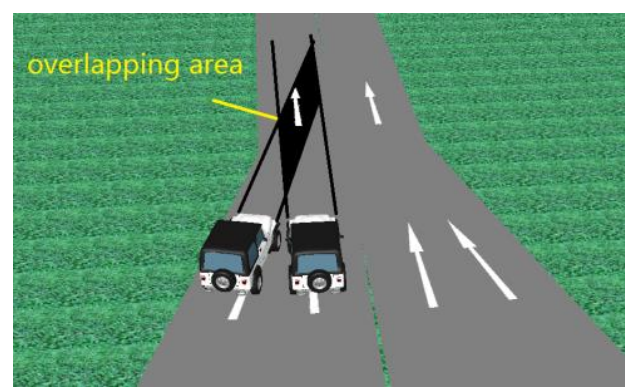

Fig. 4. Overlapping area

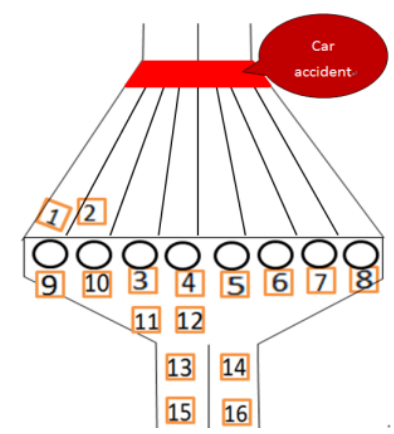

Fig. 5. Car accident

To prevent accident, we consider eliminating the overlapping area in the same group and let different groups go through the merging point continuously but not simultaneously. So we arrange the tollbooths and fan-in area as the Figure 6.

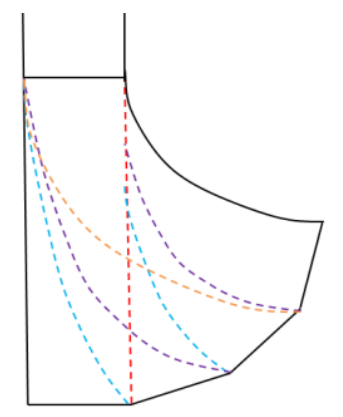

Fig. 6. Merging pattern

From the picture, we can see that vehicles in the same group will not have overlapping area benefited by paralleled path, which will reduce the probability of accident and disorder.

Based on this thought, we can get the outline of the whole barrier toll as Figure 7. The next question is how to determine the exact shape and size. 


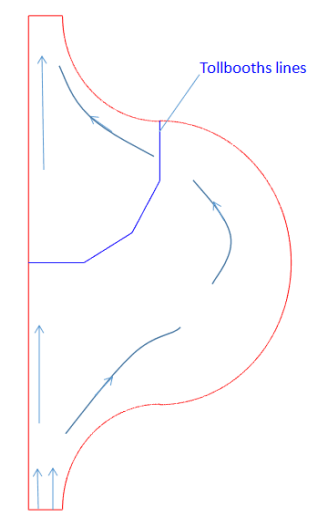

Fig. 7. The outline of the toll

\section{THE EXACT SIZE OF THE TOLL}

To ascertain the size of the toll, the cost must be taken into consideration. Thus we need to minimum the whole area of the toll. To simplify the calculation of area, we arrange the tollbooths as part of a regular polygon's sides, and establish Cartesian coordinate system with origin in the first tollbooth, which is perpendicular to the direction of traffic lanes. As we consider the direction of traffic lane is the direction of $y$ axis, then we can determine the $\mathrm{x}$ axis with origin and $\mathrm{y}$ axis. Because $B=4 \mathrm{~L}$, so we can divide the total tollbooths into 4 parts, where the tollbooths in the same group are in a line. We design each group is in the direction which have angle 0 , $\alpha, 2 \alpha, 3 \alpha$ separately between the vector in this direction and $\mathrm{x}$ axis, and the distance from the origin to the merging point is $h$. Then we design the tollbooth egress lanes as a curve, so three curves (Figure 8 curve 1, curve2, curve3) constitute the periphery of the barrier toll.

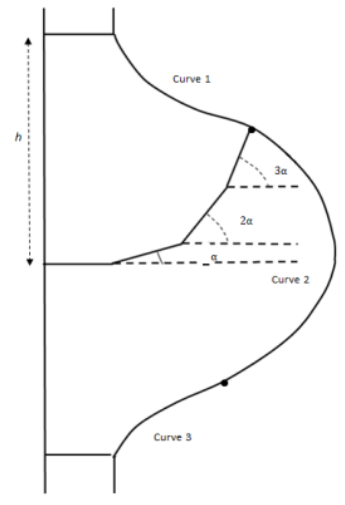

Fig. 8. The parameters of the toll

We adopt approximate treatment which is commonly used in engineering. The curve 1 is equal to a quarter of a circle, the center of curve 2 lies in somewhere of the $\mathrm{x}$ axis, and we also take a condition that vehicles will not rollover when driving along the quarter of a circle into consideration.to avoid rollover, we have a torque equilibrium equation: (Figure 9)

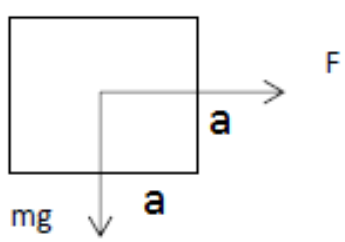

Fig. 9. Torque balance force analysis

$$
\begin{aligned}
& \mathrm{mg} * \mathrm{a}>\mathrm{F} * \mathrm{a} \\
& \mathrm{mg}>\mathrm{m} * \frac{\mathrm{v}^{2}}{\mathrm{r}}
\end{aligned}
$$

and

$$
\mathrm{v}=\frac{\mathrm{s}}{\mathrm{t} 3}=\frac{\pi \mathrm{r}}{2 \mathrm{t} 3}
$$

so we have an inequality ;

$$
\mathrm{t} 3>\sqrt{\frac{\pi^{2} * r}{4 \mathrm{~g}}}
$$

We already have $\mathrm{r}=80.92 \mathrm{~m}, \mathrm{~g}=9.8 \mathrm{~m} / \mathrm{s}$, so the conclusion is

$$
\mathrm{t} 3>4.51 \mathrm{~s}
$$

Our target is to get the smallest total area of toll plaza to ensure the minimum cost under certain constrains.

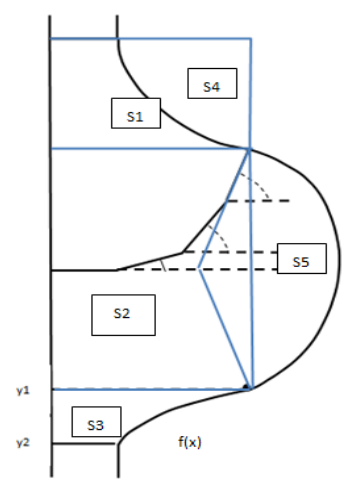

Fig. 10. The area of toll

After these assumptions and design, we can get the area of the toll plaza (Figure 2)

$$
\mathrm{s}=\mathrm{s} 1-\mathrm{s} 4+\mathrm{s} 2+\mathrm{s} 5
$$

When $\mathrm{s} 3=\int_{\mathrm{y} 1}^{\mathrm{y} 2} \mathrm{f}(\mathrm{x}) \mathrm{dy}$, so the $\mathrm{S}$ is a function of $\alpha$ and $\mathrm{h}$.

The constraint conditions are curve 1 is tangent to the traffic lane at the merging point, curve 2 is tangent to curve 1 and vehicles will not rollover while driving on the curve lanes.

Then we can determine the variables $\alpha$ and $h$ to achieve minimum area of toll plaza by using MATLAB to calculate the partial derivative of $\alpha$ and $h$, and let them be 0 simultaneously. 
Then we get $\alpha=-\frac{\pi}{6},-\mathrm{k} \pi, \frac{\pi}{6}, \mathrm{k} \pi, \frac{\pi}{4}, \frac{\mathrm{k} \pi}{2}$ and we can find a feasible solution that $\alpha=-\frac{\pi}{6}$, and $h=140.84 \mathrm{~m}$.

The result of calculation makes the toll plaza symmetric, and satisfies the condition that vehicles will not rollover, so we can get the specific plan of designing the toll plaza (Figure 11).

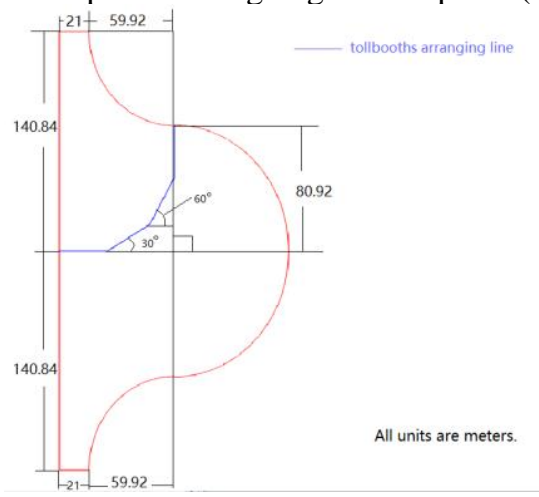

Fig. 11. The exact size of toll

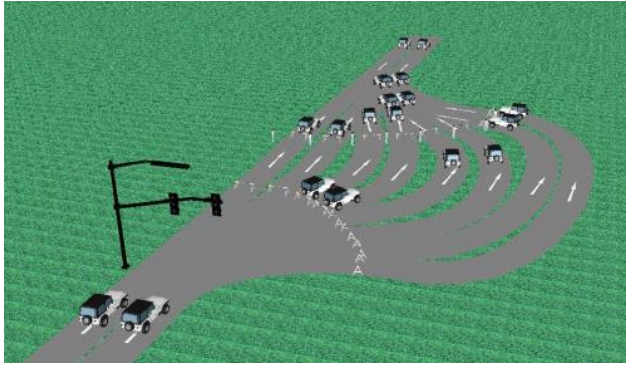

Fig. 12. Simulation of the toll

\section{A. Calculating Throughput}

In our model, the number of vehicles getting out of the barrier toll is equal to the number get in based on the theory above. So if we want to calculate number of vehicles per hour passing the merging point, we just need to count the number of vehicles entering fan-out area, which is related to the speed they adopt at that moment. To vehicles moving straight in the fan-out area, the speed is unlimited. But for those who had to turn to curved driveways, their speeds have been restricted because of centripetal force.

Considering the critical situation of the rightmost driveway, the vehicle moves along the curve 3 and curve 2 should satisfy the following equations to make sure that they will not turn over because of the centripetal force.

We consider the maximum speed which will also avoid rollover:

$$
\begin{aligned}
& \mathrm{g}=\frac{\mathrm{v} 1^{2}}{\mathrm{r} 1} \\
& \mathrm{~g}=\frac{\mathrm{v} 2^{2}}{\mathrm{r} 2}
\end{aligned}
$$

$$
\begin{aligned}
& \mathrm{v} 1=\sqrt{\mathrm{r} 1 \mathrm{~g}} \\
& \mathrm{v} 2=\sqrt{\mathrm{r} 2 \mathrm{~g}}
\end{aligned}
$$

$\mathrm{t}_{1}^{\prime}=\frac{\mathrm{k} 1}{\mathrm{v} 1}, \mathrm{t}_{2}^{\prime}=\frac{\mathrm{k} 2}{\mathrm{v} 2}(\mathrm{k} 1$ and $\mathrm{k} 2$ are the length of curve 1 and curve2)

$$
\mathrm{t}=\mathrm{t}_{1}+\mathrm{t}_{2}
$$

By this critical situation, we can get the time $t$ which is the longest time for all driveways in the lay-out area. In the ahead context, we know that we must control all vehicles' time passing fan-out area with exact the same time $\mathrm{t} 2(\mathrm{t} 2>=\mathrm{t})$. To achieve that, vehicles in straight had to sacrifice their speed. The highest speed of the straight driveway can be calculated. $\mathrm{v}_{\max }=\frac{\mathrm{h}}{\mathrm{t}}=39.27 \mathrm{~km} / \mathrm{h}$.

So to a driveway, the total amount of vehicles $\mathrm{n}=\frac{\mathrm{vt}}{1+\mathrm{D}}$.If we select $\mathrm{v}=\mathrm{v}_{\max }=39.27 \mathrm{~km} / \mathrm{h}$, average length of a vehicle add with safety distance is $54.6 \mathrm{~m}$. Then we can get the maximum amount of $\mathrm{n}_{\max }=719.23$ per hour in one driveway and the throughput $\mathrm{N}_{\max }$ of the merging point is 4315.38 for highways with 6 travel lanes. If we decrease the speed moderately, we can get average throughput $\mathrm{N} \approx 3600$ per hour with six travel lanes.

\section{CONCLUSIONS}

In this paper, we find a better solution to design the toll plaza which is creative and has not been mentioned or implemented before to solve the problem of merging after toll. Our model put significant emphasis on the main factor to the problem like waiting time, accident prevention, order and pattern to fan out and fan in, and we ignore other probable factors to simply the model. It has extensive application in the future.

\section{REFERENCES}

[1] Z.Wang, L.Kulik, and K.Ramamohanarao," Robust traffic merging strategies for sensor enabled cars using time geography," in GIS.09, Nov.2009,pp.362-371.

[2] M.Ferreira,RFermandes, H.Conceicao, W.Viriyasitavat, and O.k.Tonguz," Self-organized traffic control," in VANET 10,Sep.2010, pp.85-90.

[3] W.K.Wolterink,G.J.Heijenk, and G.Karagiannis," Constrained geocast to supportcooperative adaptive cruise control(cacc) merging." In VNC.10,Dec.2010,pp.41-48.

[4] T.Sakaguchi,A.Uno,and S.Tsugawa," Intervehicle communication for merging control system laboratory," IEEE Intell Syst,vol.18,no.4,pp.811,Jul.2003.

[5] A.Wierman," Fairness and scheduling in single sever queues," Surv.in Oper.Res. And Manag.Sci,vol.16,no.1,pp.39-48,Jan.2011.

[6] Avin C, Borokhovich M, Haddad Y, et al. Optimal virtual traffic light placement[C]//Proceedings of the 8th International Workshop on Foundations of Mobile Computing. ACM, 2012: 6.,pp.6:1-6:10.

[7] Dong H. The design of a toll plaza[C]//AIP Conference Proceedings. AIP Publishing, 2017, 1834(1): 040-043. 